\title{
Dual solutions in MHD flow on a nonlinear porous shrinking sheet in a viscous fluid
}

\author{
Fadzilah Md Ali ${ }^{1 *}$, Roslinda Nazar ${ }^{2}$, Norihan Md Arifin ${ }^{1}$ and loan Pop ${ }^{3}$
}

\section{${ }^{*}$ Correspondence:}

fadzilah@science.upm.edu.my ${ }^{1}$ Department of Mathematics \& Institute for Mathematical Research, Universiti Putra Malaysia, Serdang, Selangor 43400 UPM, Malaysia Full list of author information is available at the end of the article

\begin{abstract}
In this paper, the problem of magnetohydrodynamic (MHD) flow of a viscous fluid on a nonlinear porous shrinking sheet is studied. The boundary layer partial differential equations are first transformed into an ordinary differential equation, which is then solved numerically by the shooting method. The features of the flow for various governing parameters are presented and discussed in detail. It is found that dual solutions only exist for positive values of the controlling parameter.
\end{abstract}

MSC: $34 \mathrm{~B} 15 ; 76 \mathrm{D} 10$

Keywords: boundary layer; dual solutions; magnetohydrodynamic; shrinking sheet; numerical solution

\section{Introduction}

Boundary layer flow over a stretching sheet has been studied in various aspects since the pioneering work done by Sakiadis [1]. Lately, many researchers have studied the shrinking sheet boundary layer flow problem due to its important applications in industries which involve packaging process, for example, shrink wrapping. The study on shrinking sheet was first initiated by Wang [2] by considering the stretching deceleration surface. Miklavcic and Wang [3] proved the existence and uniqueness for viscous flow due to a shrinking sheet, and dual solutions were reported for certain range of the suction parameter. Later, Wang [4] also studied the stagnation flow towards a shrinking sheet by considering the two-dimensional and axisymmetric stagnation flows, and dual solutions were also reported only for the two-dimensional case. Further, Sajid et al. [5] and Hayat et al. [6] considered the rotating flow over a shrinking surface. The steady boundary layer flow problems induced by a shrinking sheet can be found in [7-11] in different aspects. On the other hand, the unsteady case is described in papers by Fang et al. [12] and Ali et al. [13, 14]. It is worth mentioning that dual solutions are also found in the papers by Fang [11], Fang et al. [12] and Ali et al. [14].

Recently, Nadeem and Hussain [15] solved analytically the problem of magnetohydrodynamic (MHD) flow of a viscous fluid on a nonlinear porous shrinking sheet using the homotopy analysis method and dual solutions were not reported. Hence, the present paper aims to obtain the dual solutions numerically for the problem considered in Nadeem and Hussain [15] for various controlling parameters and magnetic parameters.

(c) 2013 Ali et al.; licensee Springer. This is an Open Access article distributed under the terms of the Creative Commons Attribution License (http://creativecommons.org/licenses/by/2.0), which permits unrestricted use, distribution, and reproduction in any medium, provided the original work is properly cited. 


\section{Basic equations}

Consider the steady two-dimensional flow of an incompressible electrically conducting fluid towards a nonlinear porous shrinking sheet. The magnetic field $B(x)$ is applied normal to the shrinking and porous sheet. The governing equations of the present problem are

$$
\begin{aligned}
& \frac{\partial u}{\partial x}+\frac{\partial v}{\partial y}=0, \\
& u \frac{\partial u}{\partial x}+v \frac{\partial u}{\partial y}=v \frac{\partial^{2} u}{\partial y^{2}}-\sigma \frac{B^{2}(x)}{\rho} u,
\end{aligned}
$$

where $u$ and $v$ are the velocity components along the $x$ and $y$ directions, respectively, $v$ is the kinematic viscosity, $\rho$ is the fluid density and $\sigma$ is the fluid electrical conductivity. The boundary conditions of Eqs. (1) and (2) are

$$
\begin{aligned}
& u=-c x^{n}, \quad v=-V_{0} x^{(n-1) / 2} \quad \text { at } y=0, \\
& u=0 \quad \text { as } y \rightarrow \infty,
\end{aligned}
$$

where $V_{0}$ is the porosity of the sheet. Following Chiam [16], we assume the external electric field and polarization effects in Eq. (2) are negligible, therefore the magnetic field $B(x)$ is in the form of

$$
B(x)=B_{0} x^{(n-1) / 2} .
$$

We assume the magnetic Reynolds number is small, so that the induced magnetic field is negligible. Applying the following similarity transformations:

$$
\begin{aligned}
& u=c x^{n} f^{\prime}(\eta), \quad v=-\sqrt{\frac{c v(n+1)}{2}} x^{(n-1) / 2}\left[f(\eta)+\frac{n-1}{n+1} \eta f^{\prime}(\eta)\right], \\
& \eta=\sqrt{\frac{c(n+1)}{2 v}} x^{(n-1) / 2} y
\end{aligned}
$$

to Eqs. (1) and (2), we obtain the following ordinary differential equation:

$$
f^{\prime \prime \prime}+f^{\prime \prime}-\beta f^{\prime 2}-M f^{\prime}=0
$$

subject to the boundary conditions

$$
f(0)=s, \quad f^{\prime}(0)=-1, \quad f^{\prime}(\infty)=0,
$$

where primes denote differentiation with respect to $\eta$, while

$$
s=\frac{V_{0}}{\sqrt{\frac{c v(n+1)}{2}}}, \quad M=\frac{2 \sigma B_{0}^{2}}{\rho c(1+n)}, \quad \beta=\frac{2 n}{n+1}
$$

are the wall mass transfer (suction) parameter, the magnetic parameter and the nondimensional (controlling) parameter, respectively. 
The physical quantities of interest is the skin friction coefficient $C_{f}$ which is defined as

$$
C_{f}=\frac{2 \tau_{w}}{\rho u_{w}^{2}}
$$

where the shear stress $\tau_{w}$ is defined as

$$
\tau_{w}=\mu\left(\frac{\partial u}{\partial y}\right)_{y=0}
$$

with $\mu$ being the dynamic viscosity. Using (5) and (10), we get

$$
\operatorname{Re}_{x}^{1 / 2} C_{f}=\sqrt{2(n+1)} f^{\prime \prime}(0),
$$

where $R e_{x}=u_{w} x / v$ is the local Reynolds number.

\section{Results and discussion}

Equation (6) subject to the boundary conditions (7) has been solved numerically using the shooting method as described in the paper by Meade et al. [17]. This technique is an iterative algorithm which attempts to identify appropriate initial conditions for a related initial value problem (IVP) that provides the solution to the original boundary value problem (BVP). The results of the skin friction coefficient $f^{\prime \prime}(0)$ obtained in this study are compared with those of Nadeem and Hussain [15], as displayed in Table 1. The agreement between these results is very good. Therefore, we are confident that the present method is accurate.

Figure 1 shows the variations of the skin friction coefficient $f^{\prime \prime}(0)$ with the wall mass transfer (suction) parameter $s$ for different values of the controlling parameter $\beta$. Dual solutions are found to exist only for positive values of $\beta$. It is also found that $f^{\prime \prime}(0)$ decreases with $\beta$. On the other hand, the skin friction coefficient $f^{\prime \prime}(0)$ increases with $s$. This is because, physically, suction produces more resistance to the transport phenomena. The critical value of the suction parameter $s_{c}$ decreases as $\beta$ increases; therefore, the controlling parameter accelerates the boundary layer separation.

The variations of the skin friction coefficients with the suction parameter for $\beta=$ $0.5,1.0$ and 1.5 are displayed in Figures 2, 3 and 4, respectively. The skin friction coefficients increase with both the magnetic parameter and the suction parameter. Figures 2

Table 1 Various values of $f^{\prime \prime}(0)$ with $\beta$ when $M=2$ and $s=1$

\begin{tabular}{lll}
\hline $\boldsymbol{\beta}$ & $\begin{array}{l}\text { Nadeem and } \\
\text { Hussain [15] }\end{array}$ & Present \\
\hline 0.0 & 1.86201 & 1.86201 \\
0.1 & 1.83942 & 1.83942 \\
0.2 & 1.81648 & 1.81648 \\
0.3 & 1.79318 & 1.79317 \\
0.4 & 1.76949 & 1.76949 \\
0.5 & 1.74539 & 1.74539 \\
0.6 & 1.72087 & 1.72087 \\
0.7 & 1.69591 & 1.69590 \\
0.8 & 1.67046 & 1.67046 \\
0.9 & 1.64452 & 1.64451 \\
1.0 & 1.61804 & 1.61803 \\
\hline
\end{tabular}




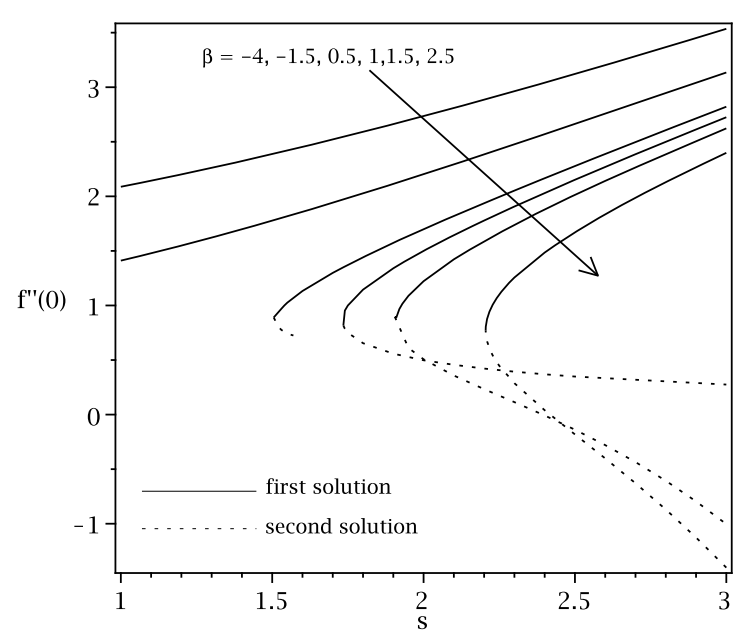

Figure 1 Variation of the skin friction coefficient for different values of $\beta$ when $M=0.25$.

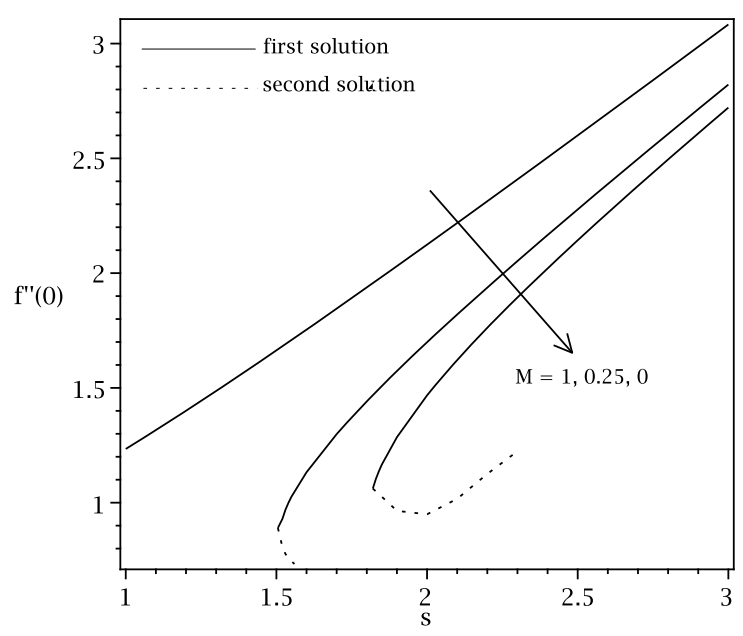

Figure 2 Variation of the skin friction coefficient for different values of $M$ when $\beta=0.5$.

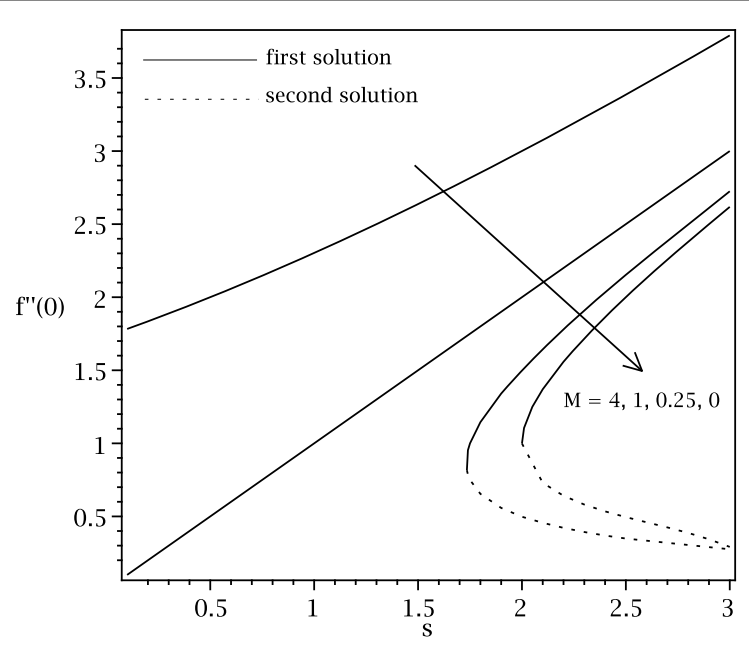

Figure 3 Variation of the skin friction coefficient for different values of $M$ when $\beta=1.0$. 


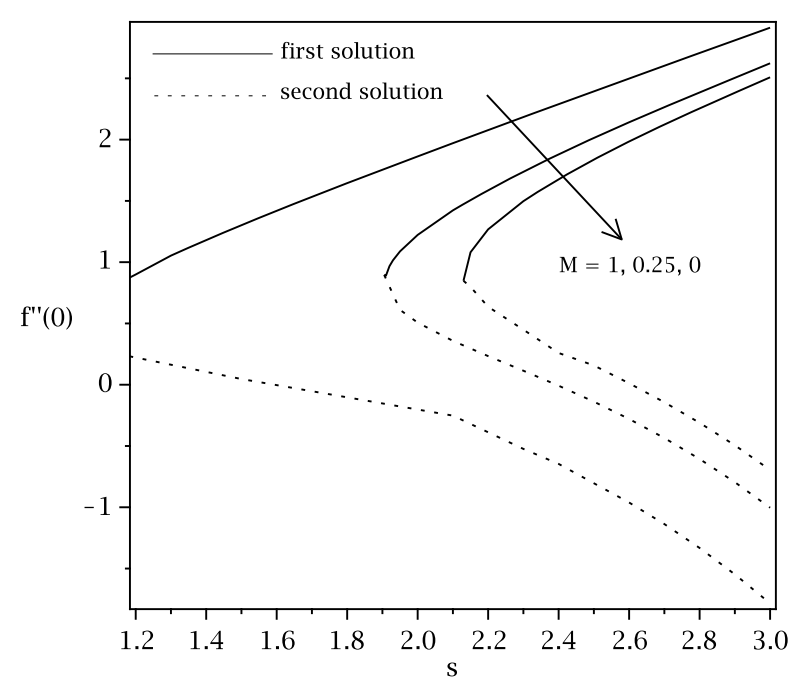

Figure 4 Variation of the skin friction coefficient for different values of $M$ when $\beta=1.5$.

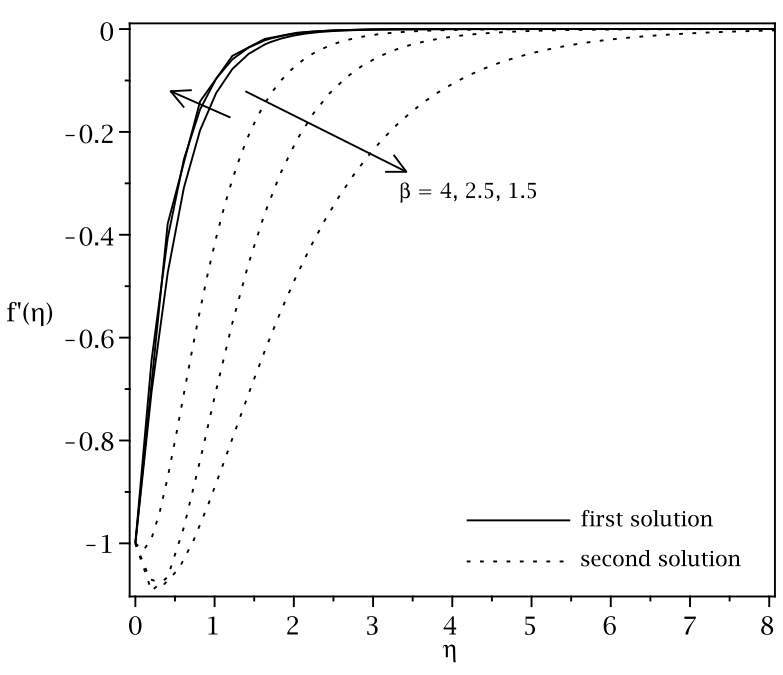

Figure 5 Velocity profiles for different values of $\beta$ when $M=0.25$ and $s=2.4$

to 4 also show the existence of the dual solutions. The boundary layer separates from the surface at $s=s_{c}$, and beyond this value, the boundary layer approximation is no longer valid. Therefore, the full Navier-Stokes equation need to be used. Normally, the first solutions are physically stable and this can be verified by performing a stability analysis; see Merkin [18] and Weidman et al. [19]. In Figures 2 to 4 , it is found that $s_{c}$ increases with $M$. Therefore, we can conclude that the magnetic parameter delays the boundary layer separation from the surface.

Figures 5 and 6 illustrate the dual velocity profiles for various $\beta$ and $M$, respectively, with both figures satisfying the boundary conditions (7). This is to prove the dual nature of the present problem. From Figure 5, the boundary layer thickness increases with $\beta$. However, the boundary layer thickness decreases as $M$ increases, as displayed in Figure 6 . 


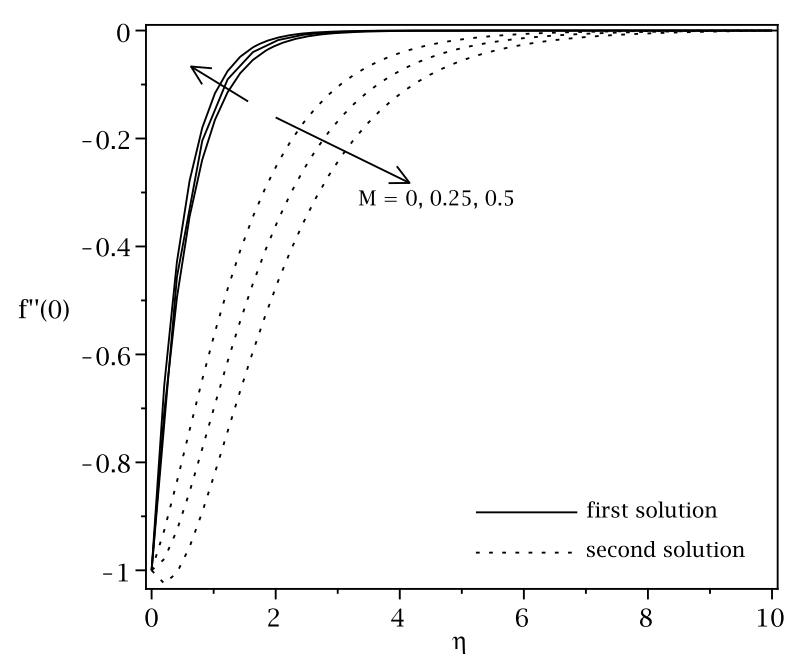

Figure 6 Velocity profiles for different values of $M$ when $\beta=1.5$ and $s=2.4$.

This phenomenon is due to the fact that $M$ creates the Lorentz force which slows down the fluid flow, hence reducing the boundary layer thickness.

\section{Conclusions}

A study is performed for the problem of MHD flow of a viscous fluid on a nonlinear porous shrinking sheet. It is observed that the dual solutions existed only for positive values of the controlling parameter. In this study, we can conclude that the controlling parameter accelerated the boundary layer separation, however, the magnetic parameter delayed the boundary layer separation.

Competing interests

The authors declare that they have no competing interests.

\section{Authors' contributions}

All authors have contributed their parts equally and have also read and approved the final manuscript.

\section{Author details}

'Department of Mathematics \& Institute for Mathematical Research, Universiti Putra Malaysia, Serdang, Selangor 43400 UPM, Malaysia. ${ }^{2}$ School of Mathematical Sciences, Faculty of Science \& Technology, Universiti Kebangsaan Malaysia, Bangi, Selangor 43600 UKM, Malaysia. ${ }^{3}$ Department of Mathematics, Babeş-Bolyai University, Cluj-Napoca, 400084, Romania.

\section{Acknowledgements}

The authors gratefully acknowledged the financial support received in the form of a FRGS research grant and a LRGS research grant (LRGS/TD/2011/UKM/ICT/03/02) from the Ministry of Higher Education, Malaysia, and DIP-2012-31 from the Universiti Kebangsaan Malaysia.

\section{Received: 21 September 2012 Accepted: 28 January 2013 Published: 18 February 2013}

\section{References}

1. Sakiadis, BC: Boundary layers on continuous solid surfaces. AlChE J. 7, 26-28 (1961)

2. Wang, CY: Liquid film on an unsteady stretching surface. Q. Appl. Math. 48(4), 601-610 (1990)

3. Miklavcic, M, Wang, CY: Viscous flow due to a shrinking sheet. Q. Appl. Math. 64, 283-290 (2006)

4. Wang, CY: Stagnation flow towards a shrinking sheet. Int. J. Non-Linear Mech. 43, 377-382 (2008)

5. Sajid, M, Javed, T, Hayat, T: MHD rotating flow of a viscous fluid over a shrinking surface. Nonlinear Dyn. 51, 259-265 (2008)

6. Hayat, T, Abbas, Z, Javed, T, Sajid, M: Three-dimensional rotating flow induced by a shrinking sheet for suction. Chaos Solitons Fractals 39, 1615-1626 (2009)

7. Fang, T, Liang, W, Lee, CF: A new solution branch for the Blasius equation - a shrinking sheet problem. Comput. Math Appl. 56, 3088-3095 (2008) 
8. Fang, T: Boundary layer flow over a shrinking sheet with power-law velocity. Int. J. Heat Mass Transf. 51, 5838-5843 (2008)

9. Fang, T, Zhang, J: Thermal boundary layers over a shrinking sheet: an analytical solution. Acta Mech. 209, 325-343 (2010)

10. Sajid, M, Hayat, T: The application of homotopy analysis method for MHD viscous flow due to a shrinking sheet. Chaos Solitons Fractals 39, 1317-1323 (2009)

11. Fang, T: Boundary layer flow over a shrinking sheet with power-law velocity. Int. J. Heat Mass Transf. 51, 5838-5843 (2008)

12. Fang, T-G, Zhang, J, Yao, S-S: Viscous flow over an unsteady shrinking sheet with mass transfer. Chin. Phys. Lett. 26 014703 (2009)

13. Ali, FM, Nazar, R, Arifin, NM, Pop, I: Unsteady shrinking sheet with mass transfer in a rotating fluid. Int. J. Numer. Methods Fluids 66, 1465-1474 (2011)

14. Ali, FM, Nazar, R, Arifin, NM, Pop, I: Unsteady flow and heat transfer past an axisymmetric permeable shrinking sheet with radiation effect. Int. J. Numer. Methods Fluids 67, 1310-1320 (2011)

15. Nadeem, S, Hussain, A: MHD flow of a viscous fluid on a nonlinear porous shrinking sheet with homotopy analysis method. Appl. Math. Mech. 30(12), 1569-1578 (2009)

16. Chiam, TC: Hydromagnetic flow over a surface stretching with a power-law velocity. Int. J. Eng. Sci. 33(3), 429-435 (1995)

17. Meade, DB, Haran, BS, White, RE: The shooting technique for the solution of two-point boundary value problems. Maple Tech. Newsl. 3, 85-93 (1996)

18. Merkin, JH: On dual solutions occurring in mixed convection in a porous medium. J. Eng. Math. 20, 171-179 (1985)

19. Weidman, PD, Kubitschek, DG, Davis, AMJ: The effect of transpiration on self-similar boundary layer flow over moving surfaces. Int. J. Eng. Sci. 44, 730-737 (2006)

doi:10.1186/1687-2770-2013-32

Cite this article as: Ali et al.: Dual solutions in MHD flow on a nonlinear porous shrinking sheet in a viscous fluid. Boundary Value Problems 2013 2013:32

\section{Submit your manuscript to a SpringerOpen ${ }^{\circ}$ journal and benefit from:}

- Convenient online submission

- Rigorous peer review

- Immediate publication on acceptance

- Open access: articles freely available online

- High visibility within the field

- Retaining the copyright to your article 